INVESTIGACIONES

\title{
Educación Física y Comunidades de Aprendizaje*
}

\author{
Physical Education and Learning Communities
}

Educação Física e Comunidades de Aprendizagem

\author{
Marta Capllonch, ${ }^{* *}$ Sara Figueras ${ }^{* * *}$ \\ *Facultad de Formación del Profesorado, Universidad de Barcelona. Paseo Vall d'Hebron, 171 (08035) de \\ Barcelona, Telf.: 93403 5141. Correo electrónico: mcapllonch@ub.edu \\ *Facultad de Psicología y Ciencias de la Educación y del Deporte Blanquerna (FPCEE), Universidad Ramon \\ Llull, Calle Císter, 34 (08022) de Barcelona. Telf.: 93 2533000. Correo electrónico: sarafc@blanquerna.url.edu
}

\begin{abstract}
RESUMEN
Las Comunidades de Aprendizaje son centros que participan de un proyecto de transformación social y cultural donde tanto la escuela como la comunidad, contribuyen a que los niños y las niñas puedan acceder, desde su propia situación, al máximo de posibilidades culturales y educativas, de manera que sus resultados educativos puedan ser iguales o superiores a los de cualquier alumno o alumna que no forme parte de un grupo en riesgo de exclusión social. El elemento fundamental es el aprendizaje dialógico basado en que todos los agentes educativos (alumnado, profesorado, familiares, voluntariado y entidades del barrio o municipio que quieran participar) disponen de espacio para el diálogo y el consenso. La educación física en estos centros participa de estos principios propiciando un clima de respeto entre los participantes, fomentando el trabajo cooperativo en grupos heterogéneos, potenciando la responsabilidad del alumnado y generando, entre otros aspectos, expectativas de éxito en los aprendizajes del alumnado.
\end{abstract}

Palabras clave: comunidades de aprendizaje, educación física, aprendizaje dialógico, grupos interactivos.

\begin{abstract}
Learning communities are schools which participate in a social and cultural transformation project where both the school and communities contribute to the boys and girls to have, from their own situation, the maximum cultural and educational opportunities. This could make the educational results to be equal or even higher than any other student that don't belong to any social exclusion risk group. The main element is the dialogic learning based on all the educational elements (students, teachers, families, volunteers and any other neighborhood and town entities which want to participate) having the same opportunity for the dialogue and agreement. Physical Education in these schools follows these standards creating a respectful climate between participants, promoting the cooperative work in heterogeneous groups, increasing student's responsibility and generating, apart from others, success expectations in the students learning.
\end{abstract}

Key words: learning communities; physical education, dialogic learning, interactive groups.

\section{RESUMO}

Comunidades de aprendizagem são centros participantes em um projeto de transformação social e cultural em que escola e comunidade contribuem com a comunidade para que as crianças possam acessar, a partir da própria situação, o máximo de possibilidades culturais e educativas, a fim de que seus resultados educacionais possam ser iguais ou superiores aos de qualquer criança que não integre um grupo em risco de exclusão social. O elemento fundamental, a aprendizagem dialógica, centra-se em todos os agentes educativos (alunos, professores, famílias,

* $\quad$ Este artículo fue solicitado por la Revista Estudios Pedagógicos en diciembre del 2011 en el contexto del proyecto de Investigación FONDECYT (Fondo de Investigación Científica y Tecnológica) N N $^{\circ} 1110016$, titulado "Educación Física y su función de transformación de las desigualdades sociales: profesorado del área y documentación ministerial”. El artículo fue aceptado en junio de 2012. 
voluntários e entidades de bairro ou município que queiram participar) dispondo espaço para diálogo e consenso. Nos centros, a Educação Física pautada nestes princípios, propicia um clima de respeito entre os participantes, potencializando, com isso, um clima de respeito entre os participantes, fomentando o trabalho cooperativo, ampliando a responsabilidade dos alunos e gerando, entre outros, expectativas de sucesso na aprendizagem destes.

Palavras chave: comunidades de aprendizagem, educação física, aprendizagem dialógica, grupos interativos.

\section{LAS COMUNIDADES DE APRENDIZAJE: UN PROYECTO DE TRANSFORMACIÓN SOCIAL Y CULTURAL DE LOS CENTROS EDUCATIVOS}

Las Comunidades de Aprendizaje surgen a partir de la noción de aprendizaje dialógico y de otras experiencias de éxito aplicadas en miles de escuelas de los Estados Unidos (Accelerated Schools, Schools Development Program, Success for All). Hoy ya se está llevando a cabo en casi un centenar de centros en España, entre Cataluña, País Vasco, Aragón, Castilla-La Mancha, Castilla-León, Andalucía, Extremadura, Murcia... y se está mostrando eficaz en la tarea de acercar la escuela a la sociedad, en la superación del fracaso escolar y en la mejora de la convivencia.

Se trata de un proyecto de éxito educativo (Gómez et al., 2010) que persigue una educación de calidad para todos y todas, respondiendo a las necesidades y retos que plantea la actual sociedad de la información, y con ello oponerse a las tendencias hacia la exclusión social que sufren los grupos más desfavorecidos (inmigrantes, minorías étnicas....). El aprendizaje depende fundamentalmente de la correlación de las interrelaciones que el niño o la niña tiene con personas de su entorno, más allá del profesorado, y en variedad de espacios, más allá de lo estrictamente escolar (Soler \& Acosta, 2005). Para ello se cuenta con la participación de la comunidad, profesorado, familiares y alumnado. Esto supone la reorganización de todo el centro, y su relación con la comunidad, barrio o pueblo, en base al diálogo:

Un proyecto de transformación social y cultural de un centro educativo y de su entorno para conseguir una sociedad de la información para todas las personas, basada en el aprendizaje dialógico, mediante una educación participativa de la comunidad, que se concreta en todos sus espacios, incluida el aula (Valls, 2000:8).

Desde esta perspectiva el proyecto de Comunidades de Aprendizaje entiende la educación como una responsabilidad compartida entre la escuela y todo el entorno del alumnado a partir del aprendizaje dialógico (Aubert et al., 2008), concepción comunicativa actual del aprendizaje que recorre las relaciones entre todos los agentes educativos del centro educativo y de la comunidad, y entre los que se establecen diálogos más igualitarios que conducen a formas de colaboración que incrementan los resultados.

Las Comunidades de Aprendizaje transforman la organización tradicional del centro educativo para optimizar todos sus recursos en vistas a que "todas las personas puedan acceder, desde su propia situación, al máximo de posibilidades culturales y educativas, de manera que sus resultados educativos puedan ser iguales o superiores" (Elboj et al., 2002: 75) a los de cualquier alumna o alumno que no forme parte de un grupo en riesgo de exclusión social.

Para reorganizar el centro se llevan a cabo actividades de formación con las familias, se rediseña la organización estableciendo comisiones mixtas de trabajo o se abre la escuela más allá del horario lectivo, abriendo espacios como la biblioteca y el aula de informática gestionados por la comunidad. Una de estas actividades son los grupos 
interactivos que nos muestran cómo los principios que orientan el proyecto toman forma en el día a día de las aulas (Flecha y Castro, 2005).

Los grupos interactivos, a los que luego se prestará más atención desde la Educación Física, representan para Flecha (2001) el aspecto más radical del proyecto de Comunidades de Aprendizaje. No se saca a nadie del aula, pero, asumiendo que el profesorado solo no puede con todas las individualidades de sus estudiantes, se introducen en el aula todos los recursos que necesita para que todos los niños y niñas aprendan y desaparezcan los problemas de fracaso y conflicto (Flecha, 2001). Las personas que entran en el aula no son sólo profesores y profesoras, sino los propios familiares de los niños y las niñas, profesorado jubilado, voluntariado, etc. Tantas personas como sean necesarias para que nadie se quede rezagado en el aprendizaje y para que todos y todas convivan desde pequeños de forma solidaria.

La presencia de todas estas personas en las aulas cuenta ya con una larga tradición, y los centros que se adhieren al proyecto de Comunidades los han ido integrando en su organización, no sin antes superar sus dudas. El Claustro del Colegio Público Ruperto Medina (1998) mencionaba: "Tuvimos muchos miedos: ver nuestra autoridad cuestionada, dar la impresión de que no nos valorábamos suficientemente, dar a entender que no teníamos respuesta para todo, sentirnos fiscalizados, no ser capaces de gobernar estas figuras procedentes del exterior, etc." Sin embargo, este claustro, como muchos otros, comprobaron que los voluntarios y voluntarias (familiares, antiguos alumnos, estudiantes universitarios en prácticas, profesorado universitario... y todas aquellas personas que quieran formar parte del proyecto), no sólo no entorpecían y descalificaban al profesorado, sino que "daban a la escuela un aire fresco, renovado y producía en nosotros un engrandecimiento interior que despertaba nuestra generosidad, nuestra capacidad de integrar" (Claustro CP Ruperto Medina, 1998: 56).

\section{TRANSFORMACIÓN DE UN CENTRO EN COMUNIDAD DE APRENDIZAJE}

El proceso de transformación de un centro escolar a una comunidad de aprendizaje parte de una reflexión profunda de toda la comunidad educativa sobre el modelo de sociedad vigente, la situación del centro respecto al territorio, el modelo de igualdad de oportunidades actual del centro, para concluir en la argumentación de la escuela que queremos para nuestras niñas y niños (CREA, 2012). Se distinguen cinco fases en el proceso de transformación y tres fases en el proceso de consolidación (Elboj et al., 2002).

\subsection{FASE DE SENSIBILIZACIÓN}

Se caracteriza por ser una fase de formación extensa que dura unas 30 horas, en la que el claustro del centro, familiares y otros miembros de la comunidad profundizan en el marco sociológico en el que se ubica el proyecto: sociedad de la información, teorías actuales de las ciencias sociales y propuestas de modelos educativos. A la vez, se explican las bases teóricas y el modelo metodológico que sustentan las comunidades de aprendizaje. Esta fase incluye también un debate y reflexión del proyecto entre los diferentes grupos de la comunidad. 
CREA $^{1}$ (2012) propone los siguientes objetivos de trabajo durante la fase de sensibilización:

a) Analizar las aplicaciones educativas en la sociedad de la información.

b) Analizar los modelos educativos de éxito que demuestran la superación del fracaso escolar con resultados muy superiores al resto de los centros educativos.

c) Analizar el proceso de transformación del centro educativo en comunidad de aprendizaje y de la implicación de la comunidad en dicho proceso.

d) Analizar las consecuencias de la desigualdad educativa.

\subsection{FASE DE TOMA DE DECISIÓN}

Es la fase donde el centro decide si iniciar o no el proceso de transformación en comunidad de aprendizaje. El proceso dura aproximadamente un mes y la decisión debe tomarse conjuntamente por toda la comunidad educativa, a saber, las familias, el claustro y la Dirección General de Educación correspondiente. Para que el acuerdo tenga validez, deben cumplirse las siguientes condiciones (CREA, 2012):

a) El $80 \%$ del claustro ha de estar de acuerdo en llevar a cabo el proyecto.

b) El equipo directivo del centro también debe estar de acuerdo.

c) La asamblea de familiares debe aprobar mayoritariamente el proyecto.

d) Debe haber un compromiso de la comunidad.

e) La Consejería de Educación deber dar apoyo a la experiencia, dotándola de un estatus propio con autonomía pedagógica y financiera suficiente para realizar el proyecto.

El objetivo de trabajo de la fase de toma de decisión se centra en decidir el inicio del proyecto (si así se decide) en base al compromiso adquirido por toda la comunidad. (CREA, 2012).

\subsection{FASE DEL SUEÑO}

Es la fase en la que se empieza el proceso de transformación. A través de reuniones por grupos, los agentes sociales del entorno discuten independientemente unos de los otros, sobre el modelo de centro educativo que se quiere para todos los niños y las niñas. Después que cada colectivo haya llegado a un consenso de sueño, se establece un proceso a través del dialogo igualitario, por el que se debe llegar a un sueño común (un modelo educativo compartido) entre todos los agentes sociales.

Finalmente, cada sueño consensuado, se contextualizará a los principios básicos de las comunidades de aprendizaje, con el objetivo de analizar el valor educativo integrador de cada elemento como motivador de la participación, y en qué modo se puede implementar en la escuela (CREA, 2012).

1 Centro Especial de Investigación en Teorías y Prácticas Superadoras de Desigualdades. 


\subsection{FASE DE SELECCIÓN DE PRIORIDADES}

Es la fase en la que se valoran las acciones y propuestas diseñadas en la fase de sueño, mesurando su viabilidad mediante un análisis de la realidad del centro educativo y de su entorno próximo. Se inicia el proceso de planificación de las propuestas, identificando y priorizando las actuaciones del proceso de transformación y diseñando una secuencia lógica de implementación de cambios.

\subsection{FASE DE PLANIFICACIÓN}

Finalmente, se activa el plan de transformación y se planifica cómo llevarlo a cabo (CREA, 2012). Esta fase puede durar dos meses y se trabaja por grupos heterogéneos de acción. Las distintas comisiones tienen que diseñar un plan de acción para cada prioridad propuesta en la fase anterior.

En el proceso de transformación resumido anteriormente, queda implicado todo el profesorado del claustro y, por tanto, todas las áreas de conocimiento. El compromiso de la educación física es, pues, formar parte activa del proceso de reflexión, creación, planificación e implementación, en el proceso de transformación en comunidad de aprendizaje. Entendemos que la educación física, en este tipo de propuestas, debe entenderse como una área totalmente integrada en la dinámica educativa del centro y comprometida en el proyecto educativo global de la escuela.

\subsection{CONSOLIDACIÓN DEL PROCESO}

Las fases anteriores tienen como objetivo poner en marcha el proyecto, mientras que las tres últimas pretenden la consolidación del proceso (Elboj et al., 2002), a saber, fase de investigación, fase de formación y fase de evaluación:

- La fase de investigación tiene como objetivo generar reflexión sobre la acción implementada, así como experimentación sobre el cambio y análisis de los resultados obtenidos con la experiencia.

- La fase de formación se refiere a la formación requerida por las distintas comisiones de trabajo en función de las necesidades generadas en el proceso de transformación.

- La fase de evaluación se caracteriza por la valoración continua del proceso de transformación del centro en comunidad de aprendizaje con la finalidad de generar correctores en las prácticas.

\section{LA EDUCACIÓN FÍSICA EN LAS COMUNIDADES DE APRENDIZAJE}

La misma definición de Comunidades de Aprendizaje nos habla de una educación participativa que se concreta en todos los espacios de la escuela y, en este sentido, entendemos que también la Educación Física debe aportar mejoras en la práctica docente, en la organización o en la apertura de la escuela a la comunidad. Por desgracia, la Educación Física ha tenido, en muchas ocasiones, un tratamiento transversal y en ocasiones ha servido como justificación para integrar al alumnado inmigrante y en riesgo de 
exclusión social, pero no como una forma de adquirir las competencias que se requieren en la sociedad de la información, sino como base de una "pedagogía de mínimos", también llamada "currículo de la felicidad", un currículum diseñado para aumentar la autoimagen de los estudiantes (Elboj et al., 2002), pero alejado de las necesidades que requiere para salir adelante.

En efecto, la Educación Física no se considera directamente vinculada al éxito de los aprendizajes escolares propiamente dicho (aprendizajes instrumentales), pero sí que podemos partir de la hipótesis de que difícilmente estudiantes poco o nada socializados, que presentan conductas agresivas, o no se sienten integrados en los centros escolares puedan llegar a tener éxito en sus estudios. En este sentido, la Educación Física, como área curricular, conjuntamente con objetivos relacionados con el desarrollo motor, el conocimiento del cuerpo o la salud corporal puede contribuir a favorecer y minimizar situaciones de exclusión social, favorecer la integración o contribuir a la resolución de conflictos potenciando valores para la paz, la solidaridad y el trabajo cooperativo, o mejora la autoestima de los niños y las niñas, todos objetivos necesarios para conseguir éxito en la escuela.

Se demuestra, desde la teoría y la práctica, que el aprendizaje dialógico de los niños, las niñas y los y las adolescentes no depende sólo de lo que ocurra dentro de las aulas, sino de la coordinación de todos aquellos espacios en los que realizan aprendizajes (Flecha, 2005: 6).

En una escuela que ha decidido realizar las transformación hacia una Comunidad de Aprendizaje "nada vuelve a ser como antes", pero este proceso de transformación no es único, ni tiene un final concreto, sino que continuamente se van buscando mejoras en los aprendizajes (Elboj et al., 2002). Por eso es tan importante replantearse la orientación y la función de la Educación Física en estos centros, pues entendemos que esta área, desde sus propios contenidos, debe contribuir a que los profesores y los estudiantes reconozcan y traten con respeto las diferentes manifestaciones culturales y proponer opciones para luchar contra las desigualdades.

La aparente distancia entre los aprendizajes instrumentales no nos ha de hacer caer en un aprendizaje de mínimos, orientada exclusivamente a cubrir unas necesidades básicas, o bien como una buena forma de desviar la atención de intolerancias y conflictos, sino en la ocasión de que cada niño y niña incrementen sus niveles de autoestima, le permita afrontar los retos escolares con éxito y aprenda a interrelacionarse cualesquiera que sea su origen o condición social.

La Educación Física constituye un área con un valioso potencial para la inclusión de los chicos y chicas inmigrantes, fundamentalmente por su carácter vivencial y por las numerosas actividades colectivas que permiten la interacción del alumnado. De esta manera, cuando la intervención educativa hace incidencia en aspectos de educación para la cooperación y la solidaridad, encuentra en la dinámica de las clases una situación propicia para la educación de las habilidades sociales, tanto para el alumnado inmigrante como para el autóctono (Capllonch et al., 2007: 62). 


\section{PRINCIPIOS DEL APRENDIZAJE DIALÓGICO APLICADOS A LA EDUCACIÓN FÍSICA}

A partir de las aportaciones de la psicología histórico-cultural, surge en la psicología de la educación el interés por profundizar en la importancia de los procesos de interacción y en el papel del diálogo en los procesos de aprendizaje. Este interés sentará las bases de teorías centradas en la importancia de la construcción social de significados a través de la interacción y el uso del lenguaje.

El aprendizaje dialógico (Flecha, 1997), basado en la concepción comunicativa y orientado a la dimensión intersubjetiva de los aprendizajes, representa un ejemplo del giro dialógico (Racionero, 2010) en las teorías del aprendizaje. Así, se entiende que la interacción es imprescindible para que se produzcan procesos de transformación individual y social a través del diálogo, ya que la construcción del conocimiento se inicia en un plano intersubjetivo /social que va concretándose en una construcción personal. El diálogo se convierte en este proceso en un elemento clave e indispensable en el aprendizaje. En este sentido se puede definir el aprendizaje dialógico como:

El que resulta de las interacciones que produce el diálogo igualitario, es decir, un diálogo en el que diferentes personas aportamos argumentos en condiciones de igualdad, para llegar a consenso, partiendo de que queremos entendernos hablando desde pretensiones de validez. (Elboj et al., 2002: 92).

Hablar desde pretensiones de validez implica que las diferentes aportaciones en un diálogo se valoran "en función de la validez de sus argumentos, en lugar de valorarlas por las posiciones de poder de quienes las realizan" (Flecha, 1997:14).

Las Comunidades de Aprendizaje se basan en los principios del aprendizaje dialógico; de esta forma, implica que todos los agentes educativos (alumnado, profesorado, familiares, voluntarios, entidades del barrio...) que deciden participar en el centro convertido en comunidad de aprendizaje tienen espacio para dialogar de manera democrática y horizontal, de manera que todas las personas tienen el mismo derecho para intervenir y decidir, en la escuela y en la clase de educación física. Para producirse un aprendizaje dialógico auténtico, no sólo es necesario que se genere un gran número de interacciones, sino que es indispensable que se produzcan unas condiciones o principios específicos en los que se debe dar el diálogo. El diálogo no debe estar marcado por relaciones de poder sino por relaciones de igualdad en la que todos y todas tengan las mismas oportunidades de aportar sus conocimientos, reconociendo de esta forma la inteligencia cultural. Por otra parte, tal como sugieren Racionero y Padrós "learners reach higher levels of learning and engage in processes of personal and social transformation when the interaction involves seven principles: egalitarian dialogue, cultural intelligence, transformation, an instrumental dimension, the creation of meaning, solidarity, and equality of differences" (2010: 151).

Así, pues, podríamos resumir los siete principios del aprendizaje dialógico (Flecha, 1997) en el contexto de la educación física, de la siguiente manera:

Dialogo igualitario: aquél en el que las diferentes aportaciones se consideran en función de la validez de los argumentos aportados y no desde criterios como la imposición de un saber culturalmente hegemónico. Este principio se refleja en situaciones en las que en las clases de educación física, se presta atención a los diferentes puntos de vista, atendiendo a los argumentos y no a la posición de las personas que los 
emiten, con la intención de llegar a consensos o de resolver conflictos: "El diálogo es igualitario cuando las aportaciones de cada participante son valoradas en función de sus argumentos (pretensiones de validez) y no en quien lo dice (pretensiones de poder)" (Elboj et al., 2002: 62).

Inteligencia cultural: es un concepto más amplio de inteligencia que los que se utilizan habitualmente, que engloba la inteligencia académica y práctica y las demás capacidades de lenguaje y acción de las personas que les permiten llegar a acuerdos. En las clases de Educación Física, encontramos un ejemplo de atención a la inteligencia cultural cuando en la evaluación se tienen en cuenta no sólo aquellos contenidos ligados a los aspectos cuantitativos del movimiento (capacidades condicionales), sino también otros aspectos vinculados a los aspectos más cualitativos (percepción, toma de decisiones...).

Transformación: este principio implica abordar las situaciones de desigualdad con vistas a transformarlas y no a reproducirlas o adaptarse a ellas. Racionero y Padrós (2010: 151) lo exponen de la siguiente forma: "Dialogic learning seeks multiple transformations: of students' level of prior knowledge, of existing knowledge and tools, of social relations, of learners themselves, and of their context of development". Cuando la Educación Física es un espacio para todo el alumnado en lugar de para sólo una parte (aquellos o aquellas más fuertes, más rápidas o más habilidosas...) se convierte en un espacio de transformación. Esto supone generar situaciones de aprendizaje en que todos y todas puedan participar y mejorar sus realizaciones independientemente de su condición social, étnica, de género o nivel de habilidad.

Dimensión instrumental: en lugar de promover un currículum de la felicidad (concretado en una serie de tareas enfocadas hacia el bienestar del alumnado, independientemente de si aprenden o no aprenden), el aprendizaje dialógico no ignora la dimensión instrumental, sino que la intensifica y la profundiza: "dialogue is not in opposition to instrumental learning" (Racionero, 2010: 63). En el caso de la Educación Física, se trataría de plantear tareas que no sólo entretengan y diviertan al alumnado, sino que impliquen también el aprendizaje de los contenidos del área curricular o incluso de otras áreas.

Creación de sentido: la separación entre la escuela, como institución, y la vida cotidiana de las personas, provoca una pérdida de sentido, de manera que se crea un abismo entre lo que la comunidad espera de la escuela y lo que la escuela es. La incorporación de referentes cercanos al alumnado (personas cercanas, personalidades con las que se sienta identificado o cualquier otro elemento que le resulte afín) en las clases de Educación Física, ayuda a acercar la clase a sus vidas cotidianas, con lo cual aumentan sus expectativas y se opera la creación de sentidos: "el sentido resurge cuando la interacción entre personas es dirigida por ellas mismas" (Elboj et al., 2002: 63).

Solidaridad: El aprendizaje dialógico se lleva a cabo de una forma solidaria. "Todos los agentes que forman parte de la comunidad educativa participan de las decisiones 
mediante sus aportaciones, democratizando la escuela a través de un diálogo igualitario y compartiendo un interés común" (Elboj et al., 2002: 63). Entendemos el principio de solidaridad como la lucha contra la exclusión social y la desigualdad, se concreta en las clases cuando el alumnado colabora con sus iguales para mejorar el aprendizaje de todas y todos. Unas veces y en ciertas disciplinas serán unas o unos los que deberán ayudar a otros u otras y en otros casos será a la inversa.

Igualdad de las diferencias: implica no entender la igualdad como homogeneización ni centrarse en la diferencia obviando la igualdad. La igualdad de las diferencias es el igual derecho de todas las personas a vivir en su diferencia: "Dialogic learning is grounded in the principle that true equality includes and equal right to differences" (Racionero y Padrós, 2010: 152). En las clases de Educación Física, este principio se tiene en cuenta cuando todo el alumnado tiene el mismo derecho a hacer clase y a aprender las mismas cosas que el resto desde su propia especificidad.

\section{LA EDUCACIÓN FÍSICA DESDE LA PERSPECTIVA DEL ALUMNADO. UN ESTUDIO DE 6 CENTROS DE COMUNIDADES DE APRENDIZAJE}

Empieza a sentirse, desde las comunidades de aprendizaje, la necesidad de orientar la práctica de la Educación Física acorde con los principios del aprendizaje dialógico, pero no existen hasta el momento evidencias que nos permitan descubrir hasta qué punto la transformación de un centro en comunidades de aprendizaje haya tenido una incidencia en la educación física. Una reciente investigación en la que hemos estado trabajando estos últimos cuatro años (Capllonch, 2008-2011) nos ha permitido hacer una radiografía de cómo el profesorado está orientando el área para contribuir al éxito de todos los niños y niñas. Este proyecto pretendía identificar los conflictos que se producían en la Educación Física y potenciar los mecanismos necesarios para su resolución, utilizando la Educación Física y el Deporte como un espacio de convivencia. La elección de realizarse en Comunidades de Aprendizaje, respondía, por un lado, a la numerosa presencia de alumnado inmigrante en una misma aula, y por otro, a las dinámicas de estos centros de afrontar el conflicto mediante la participación y el diálogo igualitario de todos los miembros de la comunidad educativa (profesorado, alumnado, familiares de cualquier cultura...).

La investigación de orientación comunicativa ${ }^{2}$ contaba con técnicas de recogida de información tanto cuantitativas como cualitativas. De ella, tomamos la parte de los cuestionarios administrados al alumnado de $5^{\circ}$ y $6^{\circ}$ de primaria de los centros participantes en la investigación (3 Comunidades de Aprendizaje de Cataluña y 3 del País Vasco), cuyas preguntas estaban directamente vinculadas a la percepción sobre el funcionamiento de las clases de Educación Física. Cada una de ellas iba orientada a comprobar en qué medida se aplicaban o se asumían los principios del aprendizaje dialógico desde las aulas de

2 Se trata de una metodología con una importante base teórica y práctica. A nivel teórico incorpora nociones y conceptos de autores como Beck y Habermas, y a nivel práctico ha sido reconocida por el Programa Marco de Investigación Europea en el estudio de las desigualdades sociales para su posterior transformación. Esta metodología ha sido empleada en diversos proyectos de investigación y desarrollo a nivel nacional e internacional. 
Educación Física mediante cuestiones que permitieran describir el clima de las clases, las estrategias que se llevaban a cabo, las formas de organización, el tratamiento de los contenidos y la orientación de las actividades, el nivel de responsabilidad del alumnado en su aprendizaje, la presencia del diálogo y las expectativas de éxito de los aprendizajes.

Los resultados obtenidos nos permite extraer que en las Comunidades de Aprendizaje estudiadas la Educación Física se orienta a:

Propiciar un clima de respeto entre los participantes: Una de las preguntas del cuestionario iba orientada a conocer qué cosas sucedían en las clases de Educación Física. En las opciones de respuesta aparecían alternativas como si los niños se burlaban o se separaban de las niñas o al revés, si el profesor o profesora separaba a los niños de las niñas, si las personas con mayores habilidades se burlaban o se separaban de aquellas a las que no se les daba bien, si el profesor o la profesora separaba a los niños según los diferentes niveles de habilidad, si algunas personas sólo jugaban para ganar y había problemas cuando no ganaban, si las trampas les hacían enfadar o si se realizaban juegos en los que se eliminaban a las personas.

Todas estas cuestiones tenían por objetivo tener una idea sobre cómo se sentía el alumnado en el aula, qué tipología de actividades se llevaban a cabo, o las estrategias que utilizaba el profesorado para organizar las actividades. El análisis de los datos permite asegurar que, mayoritariamente en las Comunidades de Aprendizaje estudiadas, se trabaja en un clima de respeto, donde no se dan las burlas entre niños y niñas, se respetan las diferencias individuales y los diferentes niveles de habilidad y donde jamás se separa a los niños de las niñas. Asimismo, el profesorado evita situaciones de confrontación mediante actividades en las que se propicia la participación de todos y todas sin eliminar a nadie, para evitar al máximo que no se respeten las reglas o se hagan trampas para ganar.

Y es que, en efecto para algunos autores como Ortí (2003), los conflictos más destacados en Educación Física están relacionados con la discriminación por razones de género, o de habilidad (competencia motriz), con una búsqueda excesiva de la victoria, o bien con estereotipos culturales.

Fomentar el trabajo cooperativo en grupos heterogéneos: Uno de los aspectos en los que se fundamenta el aprendizaje dialógico es en la interacción entre las personas. Para saber en qué medida en las clases de educación física se daban situaciones de cooperación, se preguntó exactamente a los niños y niñas: "en Educación Física, ¿tenéis momentos donde las personas que más saben ayudan y trabajan junto con las que menos saben?". Las opciones de respuesta se distribuían, en "sí, cada día"; "sí, bastante, cada semana"; "sí, a menudo" y, finalmente, "no, nunca". Pues bien, en las Comunidades de Aprendizaje analizadas, la suma de las frecuencias de que las personas que más saben ayudan y trabajan con las que menos saben cada día, cada semana o a menudo, alcanza casi el 90\%, lo que sugiere que, en efecto, desde la Educación Física el componente de cooperación y ayuda mutua entre iguales es muy alto.

El aprendizaje cooperativo es aquella metodología educativa que se basa en el trabajo en pequeños grupos, generalmente heterogéneos, donde los alumnos trabajan juntos para mejorar su propio aprendizaje y de los otros (Velázquez Callado, 2003, 2004). Según los 
estudios del mismo autor, la metodología cooperativa en Educación Física, aunque todavía innovadora, ya da muestras de la posibilidad que ofrece al desarrollo de habilidades sociales y posibilita a todos los alumnos alcanzar el éxito mediante la práctica motriz que a la vez repercute en un incremento de la autoestima. Además, como nos recuerdan López Pastor, García de la Puente e Iglesias (2004), en aquellos contextos educativos con un marcado índice de inmigración, la utilización de modelos de Educación Física más habituales, basados en juegos competitivos y eliminatorios, dificultan enormemente la integración y suelen generar todavía más conductas de exclusión, marginación, enfrentamiento, conflicto y agresiones. En cambio, la utilización de modelos de Educación Física que tienen como principal finalidad la convivencia y la integración, basados en enfoques metodológicos cooperativos han demostrado ser eficaces a la hora de superar situaciones de marginación, conflicto y avanzar hacia una integración real de todo el alumnado del grupo clase.

Se observa que en las comunidades de aprendizaje estudiadas, la organización de la clase se estructura básicamente en grupos heterogéneos tanto de niños y niñas, con diferentes niveles de habilidad, y donde los que más saben tienen posibilidad de ayudar y trabajar con los que menos saben para conseguir un mismo objetivo. Es decir, colaborar con sus iguales para mejorar el aprendizaje de todos y todas.

Promover la responsabilidad entre el alumnado: Otra de las cuestiones planteadas al alumnado era que describiera en qué nivel se colocaría en relación a una serie de cuestiones vinculadas con la responsabilidad. Éstas eran: "si pasaba de todo y no dejaba trabajar a nadie"; "si pasaba de todo, pero dejaba trabajar"; "si hacía las cosas tan sólo cuando estaba presente el profesor"; "si las hacía independientemente de que el profesor estuviera presente"; y, finalmente, "si hacía las cosas solicitadas y además ayudaba a los demás". La tendencia de respuesta mayoritariamente observada era que el alumnado de las Comunidades de Aprendizaje estudiadas tendía a realizar las tareas de manera autónoma y ayudando a los compañeros y compañeras que tenían mayores dificultades en realizarlas.

Este aspecto es fundamental para que el alumnado se responsabilice de su propio aprendizaje. No podemos olvidar, además, que la actividad física ofrece un contexto natural ideal para el desarrollo de situaciones e interacciones que destapan las habilidades personales y sociales de los participantes, unas habilidades que resultan difícilmente observables en otros contextos educativos como el aula (Escartí et al., 2005). También la metodología cooperativa contribuye a que el alumnado relacione directamente su valoración de la clase, con la responsabilidad que adquiere a través de ésta (Fernández-Río, 2000; González de Mesa y Fernández-Río, 2004).

Impulsar el diálogo: Vinculado con el papel del diálogo en el aprendizaje, otra de las cuestiones iba orientada a conocer la presencia o ausencia de diálogo para tratar temas referentes a la asignatura. Para ello se preguntó al alumnado si en Educación Física había momentos en los que el profesorado y el alumnado se ponían de acuerdo en las cosas que hacían referencia a la clase. Entre las opciones de respuesta aparecía una escala de tres posibilidades que iban desde "sí, el profesor o la profesora nos pregunta y hablamos mucho", casi cada día, cada semana o de vez en cuando. Finalmente, otra 
opción era que "no, el profesor o profesora no nos pregunta y no solemos hablar". Los resultados obtenidos reflejan que en las comunidades de aprendizaje estudiadas, el profesorado y el alumnado a menudo hablan y se ponen de acuerdo en cuestiones que hacen referencia a las clases de Educación Física: espacios a utilizar, las normas del juego, las actividades a realizar... prestando atención a los diferentes puntos de vista, y atendiendo las opiniones de todos los niños y niñas, no sólo del profesorado.

El papel del diálogo en las clases de Educación Física ya ha sido mencionado por diferentes autores: Fernández Balboa y Marshall (1994), Tormos et al. (2003), Ruiz Omeñaca (2004), etc., para situaciones tan variadas como la negociación, el tratamiento del conflicto, la participación del alumnado en la toma de decisiones... y resulta fundamental para que el alumnado se implique en su propio aprendizaje y tenga consciencia del papel que ocupa en el grupo y de su responsabilidad.

Generar expectativas de éxito de los aprendizajes: Para comprobar en qué medida la clase de Educación Física era un espacio de transformación en el que se generaban situaciones de aprendizaje en que todos y todas podían participar y mejorar, independientemente de su nivel de partida, se planteó la pregunta de si en Educación Física todo el mundo podía sacar buenas notas. Las opciones de respuesta eran 2. Una en la que se decía que "no, casi siempre son las mismas personas (las más fuertes, las más rápidas...) las que sacan buenas notas en todo lo que hacemos en Educación Física”. La otra opción decía que "sí, en Educación Física no sólo se trata de saltar mucho o correr muy rápido, también se valoran otras cosas”. Cerca del $80 \%$ del alumnado mencionó que en sus clases de Educación Física todo el mundo podía sacar buenas notas, independientemente de su nivel de habilidad motriz, ya que no sólo se valoraba el nivel de ejecución, sino también aspectos como el comportamiento, la toma de decisiones, la ayuda a los compañeros, entre otras cosas.

Vinculado con el nivel de éxito, y sobre todo para comprobar de qué manera la clase de Educación Física trataba la igualdad de las diferencias, se solicitó a los niños y niñas que finalizaran la frase siguiente: "Cuando se hace una cosa nueva en Educación Física, una parte del alumnado - al que se le da bien- la aprende rápido..." Las opciones de respuesta eran: “... y el resto no"; “... y del resto, que tardan un poco más, sólo algunos la aprenden”; y, finalmente, “... y del resto, que tardan un poco más, todos o la mayoría la aprenden". En este caso, igual que en el anterior, la percepción mayoritaria del alumnado era que en las clases de Educación Física todos o la mayoría podían aprender, es decir, que no sólo se aseguraba la participación, sino la mejora de las realizaciones independientemente de su condición social, étnica, de género o nivel de habilidad.

Hemos comprobado que, para ello, los maestros y maestras utilizan estrategias en las que es imprescindible tener en cuenta que los niños y niñas aprendan cosas nuevas, vinculadas a sus intereses $\mathrm{y}$, además, de buena manera. 


\section{LOS GRUPOS INTERACTIVOS EN EDUCACIÓN FÍSICA}

Al menos en dos de las comunidades de aprendizaje participantes en la investigación Juega, Dialoga y Resuelve (2008-2011), han tenido o siguen manteniendo iniciativas vinculadas con la organización de grupos interactivos en las clases de Educación Física. Para ello y dependiendo del número de personas voluntarias y del tipo de actividad a desarrollar se divide la clase en 2 ó 3 ó 4 grupos con la única condición de que éstos sean heterogéneos, tanto en cuestión de género como nivel de aprendizaje y origen cultural. Se preparan actividades de 20 ó 30 minutos de duración, lo suficiente como para poder desarrollar la actividad y mantener el nivel de atención y motivación. Pasado este tiempo, el grupo cambia de actividad y de tutor o tutora, con lo que la clase se desarrolla de una manera más dinámica y entretenida.

En uno de los centros, que contaba con una baja matrícula de alumnado, juntaban para la clase de Educación Física de dos horas de duración los grupos de $5^{\circ}$ y $6^{\circ}$ de primaria. Las personas adultas en el aula eran: la profesora de Educación Física, el profesor tutor que quedaba libre (al hacer la clase conjuntamente $5^{\circ}$ y $6^{\circ}$ ), la asistente social del centro y una persona voluntaria profesora de la Universidad de Barcelona. De esta manera, se podía trabajar o bien en 4 grupos, cada uno de ellos con un adulto, para contenidos como las habilidades y destrezas básicas o específicas que implicaban cierto riesgo, y que una persona sola adulta no puede asumir con un grupo numeroso; o bien en dos grupos, con dos adultos cada uno para actividades y juegos deportivos como el Pichi (variante escolar del baseball) o Korfball (variante del baloncesto). En este caso, cada uno de los adultos formaba parte de uno de los equipos. De esta manera, como mencionan Flecha y Castro (2005), desaparecen los problemas de disciplina en el aula, incrementa la motivación por el aprendizaje y se crea una solidaridad desde la base.

La participación de las personas voluntarias en el aula de Educación Física, en colaboración con el docente, ha de ir orientada a que se produzcan interacciones entre iguales lo más ricas posibles, prestar mayor atención al desarrollo del trabajo de los niños y las niñas, identificar dificultades e intentar que sean los propios niños y niñas los que lleguen a resolverlos fomentando así la cooperación y desarrollando la autoestima. El docente, por su parte, adopta un nuevo papel de dinamizador y coordinador de las actividades que se llevan a cabo en el aula.:

Al mejorar la práctica con la participación de diferentes agentes aumenta el apoyo social para destinar más recursos a la educación y más profesorado para las aulas. Su voluntariado no sustituye profesorado, sino que lo complementa y crea condiciones sociales para su aumento (Puigvert y Santacruz, 2006: 171).

En los grupos interactivos se da un importante valor a las tareas relacionadas con el lenguaje, la expresión, el razonamiento y la resolución de problemas. Así, en las clases de Educación Física no es de extrañar ver a niños o a niñas presentando un juego de su país de origen, proponiendo variantes de una actividad, explicando las normas de juego, o revelando a algún compañero o compañera algún truco para llevar a cabo alguna habilidad. Y es que en los grupos interactivos el alumnado puede enseñar y en otros momentos aprender de sus compañeros y compañeras. De esta manera el alumnado se siente partícipe y contribuye para mejorar la cooperación entre el grupo. Y es que en efecto, la dinámica que se genera en el grupo asegura que todos los niños y niñas se 
sientan responsables del propio aprendizaje, así como del aprendizaje de sus compañeros y compañeras (Elboj et al., 2002).

En los grupos interactivos multiculturales el alumnado con un nivel más avanzado ayuda a los compañeros y compañeras que presentan más dificultades. Así, además de aprender en solidaridad a compartir, ayudarse y respetarse con niños y niñas de otras culturas, consolidan y amplían sus conocimientos y se promueve también de este modo la adquisición de las habilidades comunicativas necesarias para el diálogo y el trabajo en equipo, muy valorado en el mercado de trabajo de la actual sociedad de la información (Flecha \& Castro, 2005).

En una de las escuelas percibimos, a la hora del recreo en el patio, una piña de niños y niñas discutiendo entre ellos y alrededor de una voluntaria. Ocurría que la maestra de Educación Física había dado la consigna de que no podrían participar de las actividades de la clase si no dominaban las tablas de multiplicar. El espacio de juego se llenó de niños y niñas preguntándose y ayudándose los unos a los otros con las tabla del 7, del 8 y del 9.

Con los grupos interactivos se consigue, además, mantener unido al grupo-clase sin segregar al alumnado que se considera de diferente nivel, conflictivo o que presenta más dificultades. En lugar de sacar del aula a estos niños y niñas, ponemos en el aula los recursos necesarios para que este alumnado pueda seguir la clase con sus compañeros y compañeros. Y es que, en efecto, los grupos interactivos van en contra de basar la cotidianeidad del centro en prácticas segregadoras y organizar una vez al año una campaña de solidaridad. Los grupos interactivos mejoran al mismo tiempo el aprendizaje instrumental y la solidaridad como partes de un mismo proceso, y mencionan que la igualdad a la que se avanza no es una igualdad por debajo, que retrasa a unos para poder adelantar más a otros, sino que supone la aceleración del aprendizaje para todos y todas (Puigvert y Santacruz, 2006).

Un estudiante del Master de Actividad Motriz y Educación de la Universidad de Barcelona que actuaba de voluntario en grupos interactivos de Educación Física mencionó tras la experiencia:

Se deben aprovechar las fortalezas de la Educación Física que conjuntamente con los grupos interactivos en el área puede ayudar a mejorar algunos aspectos relacionados con la inclusión, la socialización, la conflictividad, la comunicación y el diálogo, la asimilación de normas y reglas, la autoestima, el trabajo cooperativo, los aprendizajes, etc. Y con ello contribuir a la mejora de toda la comunidad educativa si se gestiona adecuadamente y nos implicamos en trasladar esta experiencia a todos los espacios del centro (Galobardes, 2010).

Tras la intervención, recopilación y análisis de los datos de su experiencia como voluntario, este estudiante define que en Educación Física los grupos interactivos posibilitan:

- Disminuir el grado de marginación del alumnado con riesgo de exclusión social,

- Aumentar el control del grupo y en consecuencia mejorar la atención y la parti cipación del alumnado.

- Afianzar el sentimiento de protagonismo de los niños y las niñas.

- Incrementar los vínculos emocionales y afectivos propios de la socialización y la interacción.

- Mejorar la aceptación de normas y reglas que se traspasan fuera del aula de Educación Física.

- Favorecer la comunicación, el diálogo y la expresión entre el alumnado y entre 
el tutor o tutora y el alumnado.

- Disminuir el grado de conflictividad.

- Acelerar los procesos de aprendizaje (Galobardes, 2010).

\section{CONCLUSIONES}

Orientar adecuadamente la Educación Física empieza a ser una demanda en las Comunidades de Aprendizaje que ya llevan una trayectoria de éxito y que quieran ampliarla a todos los espacios del centro. Una reciente investigación vinculada a la prevención y resolución de conflictos desde la Educación Física en las Comunidades de Aprendizaje, nos ha permitido conocer la percepción del alumnado sobre el trabajo que se desarrolla en las aulas, y con ello comprobar cómo se lleva a cabo la apropiación del aprendizaje dialógico desde el área.

De los resultados extraídos de los cuestionarios administrados al alumnado de $5^{\circ}$ y $6^{\circ}$ de primaria de 6 Comunidades de Aprendizaje estudiadas, podemos decir que en estos centros la Educación Física propicia un clima de respeto entre los participantes, sea cualquiera que sea su género, etnia o nivel de habilidad. Se fomenta el trabajo cooperativo en grupos heterogéneos; y han empezado a surgir experiencias vinculadas con grupos interactivos, donde los que más saben pueden ayudar a los que menos saben a conseguir metas comunes. Se promueve la responsabilidad entre el alumnado, para que participe en su propio aprendizaje y en el de sus compañeros y compañeras. Se impulsa el diálogo entre todos y todas independientemente del nivel que ocupa en el grupo, y de todos los aspectos que hacen referencia al aula. Finalmente, se generan expectativas de éxito de los aprendizajes donde no sólo cuenta la participación sino también los aprendizajes independientemente del nivel de partida.

Todavía será necesario realizar más investigación, y avanzar en el camino de experiencias que avalen el éxito para todos y todas. La Educación Física, independientemente del papel que juegue en la escuela, debe contribuir eficazmente a conseguir una sociedad de la información para todos y todas.

\section{REFERENCIAS BIBLIOGRÁFICAS}

Aubert, A., Flecha, A., García Yeste, C., Flecha, R., y Racionero, S. (2008). Aprendizaje dialógico en la Sociedad de la Información. Barcelona: Hipatia.

Capllonch, M. (2008-2011). Juega Dialoga y Resuelve. La superación de conflictos en educación física mediante el modelo comunitario. Diseño de un programa específico para comunidades de aprendizaje, con referencia: SEJ2007-61757/EDUC. Plan Nacional I+D+i (2007-6200).

Capllonch, M., Lleixà, T. y Godall, T. (2007). El professorat d'Educació Física a l'escola multicultural. Percepcions del context i necessitats de formació. Revista Temps d'Educació, n.33, 61-74.

Claustro CP Ruperto Medina. (1998). El voluntariado en la Comunidad de Aprendizaje de Ruperto Medina. Aula de Innovación Educativa, n.72, 56-57.

CREA. (2012). Fases de transformación de un centro en comunidades de aprendizaje. Extraído el 22 de marzo de http://utopiadream.info/ca/?page_id=12

Elboj, C., Puigdellívol, I., Soler, M., y Valls, R. (2002). Comunidades de aprendizaje. Barcelona: Graó. 
Escartí, A. y Pascual, C. y Gutiérrez, M. (2005). Responsabilidad Personal y Social a través de la educación física y el deporte. Barcelona: Graó.

Fernández-Balboa, J. y Marshall, J. (1994). Dialogical Pedagogy in Teacher Education: Toward an Education for Democracy". Journal of Teacher Education, vol.45, n.3, 176-182.

Fernández-Río, J. (2000). La metodología cooperativa como herramienta para la enseñanza de las habilidades motrices básicas en educación física. Tándem. Didáctica de la Educación Física, n.1, 107-117.

Flecha, R. (1997). Compartiendo palabras. El aprendizaje de las personas adultas a través del diálogo. Barcelona: Paidós.

Flecha, R. (2001). Las comunidades de aprendizaje como expertas en resolución de conflictos. Mesa redonda sobre La convivencia en los centros escolares. Experiencia de como construir la convivencia. En Seminario sobre la convivencia en los centros escolares como factor de calidad. Construir la convivencia, Madrid, 21 y 22 de febrero de 2001. Madrid: Consejo Escolar del Estado.

Flecha, R. (2005). Prólogo. En C. Elboj, I. Puigdellívol, M. Soler \& R. Valls (Eds.), Comunidades de aprendizaje. Transformar la educación (pp. 5-7). Barcelona: Graó.

Flecha, R., y Castro, M. (2005). Igualdad de diferencias en la escuela multicultural: Las Comunidades de Aprendizaje. En R. Bielsa, A. Rivero, J. J. Molina \& A. Manzano (Eds.), Actas del Congreso Internacional UEM. Actividad Física y deporte en la sociedad del siglo XXI. Madrid: Universidad Europea de Madrid.

Galobardes, E. (2010). Els grups interactius en Educació Física en el context de la Comunitats d'Aprenentatge. Barcelona: Universidad de Barcelona.

Gómez, A. y Mello, R. y Santa Cruz, I. y Sordé, T. (2010). De las experiencias de Comunidades de Aprendizaje a las políticas basadas en sus éxitos. Revista Interuniversitaria de Formación del Profesorado, Vol. 67, n. 24.1, 113-126.

González de Mesa, C., y Fernández-Río, J. (2004). La investigación-acción: perspectiva innovadora en educación física. Tándem. Didáctica de la Educación Física, n.15, 58-67.

López Pastor, V., García de la Puente, J. M., Iglesias, P. (2004). El alumnado inmigrante en el área de Educación Física: el trabajo de un seminario sobre esta temática en la provincia de Segovia. En Actas del XXII Congreso Nacional de Educación Física. La formación inicial del profesorado ante el reto europeo. La Coruña: Universidad de La Coruña.

Ortí, J. (2003). La resolución de conflictos en la Educación Física. Tándem. Didáctica de la Educación Física, n.13, 40-50.

Puigvert, L., \& Santacruz, I. (2006). La transformación de centros educativos en comunidades de aprendizaje. Calidad para todas y todos. Revista de Educación, n.339, 169-176.

Racionero, S. (2010). Egalitarian dialogue and instrumental dimension. Two principles of dialogic learning in the classroom. Psychology, Society and Education, vol.2, n.1, 61-70.

Racionero, S. y Padrós, M. (2010). The dialogic turn in educational psychologoly. Revista de Psicodidáctica, vol.15, n.2, 143-162.

Ruiz Omeñaca, J. V. (Ed.). (2004). Pedagogía de los valores en la Educación Física. Madrid: CCS.

Soler, M., y Acosta, S. (2005, 1 i 2 d'abril). Comunidades de aprendizaje: propuesta educativa igualitaria en la sociedad de la información. Artículo presentado en Jornadas Arte y educación en la sociedad de la información, Barcelona: Fundació La Caixa.

Tormos, A., Armenteros, I., Sanfrancisco, R., Ruíz, R. M., Fornes, M. y Merino, M. (2003). Conflictos cotidianos abordados desde una perspectiva humana y social: una oportunidad para Diego y Silvia. Tándem. Didáctica de la Educación Física, n.13, 63-74.

Valls, R. (2000). Comunidades de Aprendizaje. Una práctica educativa de aprendizaje dialógico para la sociedad de la información. Universidad de Barcelona: Barcelona. 
Velázquez Callado, C. (2003). El aprendizaje cooperativo en Educación Física: qué, para qué, porqué y cómo. En Actas del III Congreso Estatal y I Iberoamericano de Actividades Físicas Cooperativas. Ampliando horizontes a la cooperación. Valladolid: La Peonza.

Velázquez Callado, C. (2004). Las actividades físicas cooperativas. Una propuesta para la formación en valores a través de la educación física en las escuelas de educación básica. México: Secretaría de Educación Pública. 
